\title{
The Degree of Retinopathy Correlates with the Presence of Silent Myocardial Ischemia in Diabetic Patients
}

\author{
Ehab El-Hefny ${ }^{1,}$, , Abdel Mageed Tag El-Din ${ }^{1}$, Ayman Sadek $^{1}$, Eslam Abbas ${ }^{2, ~ *}$ \\ ${ }^{1}$ Faculty of Medicine, Al Azhar University, Cairo, Egypt \\ ${ }^{2}$ Oncocardiology Department, Dar El Salam Cancer Center, Cairo, Egypt
}

Email address:

ehabelhefny@hotmail.com (E. El-Hefny), islam.omr@med.au.edu.eg (E. Abbas)

${ }^{*}$ Corresponding author

\section{To cite this article:}

Ehab El-Hefny, Abdel Mageed Tag El-Din, Ayman Sadek, Eslam Abbas. The Degree of Retinopathy Correlates with the Presence of Silent Myocardial Ischemia in Diabetic Patients. Cardiology and Cardiovascular Research. Vol. 4, No. 2, 2020, pp. 34-46.

doi: $10.11648 /$ j.ccr.20200402.12

Received: February 28, 2020; Accepted: March 16, 2020; Published: April 21, 2020

\begin{abstract}
Background: Silent myocardial ischemia is a major component of the total ischemic burden for patients with ischemic heart disease. The disease is more prevalent in diabetic patients than their peers, and early detection of the high-risk group would play an integral role in the prevention of sudden cardiovascular accidents that are common in these patients. Methods: A prospective cohort study including asymptomatic 53 diabetic patients with diabetic retinopathy who suffer no ischemic cardiac symptoms was conducted. The presence and degree of diabetic retinopathy were evidenced using fundus examination and optical coherence tomography. All the enrolled patients underwent stress-resting 99mTc SestaMIBI myocardial perfusion scintigraphy to detect the ischemic burden. The relation between diabetic retinopathy and silent myocardial ischemia was stratified using statistical analysis. Results: A total of 13 diabetic patients $(24.5 \%)$ have silent myocardial ischemia in the form of regional myocardial perfusion abnormalities. The strongest predictors of abnormal tests were the presence of moderate to severe retinopathy, comorbid hypertension and diabetic duration for more than 10 years. Conclusion: Silent myocardial ischemia affects one in four asymptomatic diabetic patients suffering from diabetic retinopathy. The presence of comorbid risk factors such as high-grade retinopathy, hypertension and/or long diabetic duration surge the incidence and considered additional predictors of the disease.
\end{abstract}

Keywords: Diabetic Retinopathy, Silent Myocardial Ischemia, Optical Coherence Tomography, Myocardial Perfusion Imaging

\section{Introduction}

Coronary heart disease (CHD) is a leading cause of mortality in individuals with diabetes [1, 2]. While the macrovascular disease is the primary pathogenic mechanism underlying CHD in the general population, the microvascular disease may play a prominent role in CHD development in diabetic individuals [3-5]. Myocardial ischemia in patients with diabetes is often asymptomatic and frequently in an advanced stage when it becomes clinically manifest $[6,7]$. Once $\mathrm{CAD}$ is symptomatic in diabetes, morbidity and mortality are high and significantly worse than patients without diabetes, which -in turn- highlights the significance of allocation of the high-risk group and subsequent early diagnosis of the disease.

Earlier studies have shown that diabetic retinopathy predicts cardiovascular mortality and morbidity in both Type 1 [8-11] and Type 2 [12-16] diabetic populations. Furthermore, the findings of recent studies also suggest that retinopathy is an early sign of other vascular complications and is strongly associated with the development of CAD [1, $5,17]$. Additional studies reported that diabetic patients have a higher prevalence of silent myocardial ischemia and asymptomatic coronary heart disease [18, 19]. However, information on the impact of the degree of diabetic retinopathy on the incidence of silent myocardial ischemia in diabetic populations is limited, especially with respect to the impact of other relevant comorbid risk factors such as age, sex, hypertension, and duration of diabetes. 
In this regard, an interesting question, that arises from a clinical perspective, is whether screening for silent myocardial ischemia should be conducted for asymptomatic patients with diabetic retinopathy, and how to allocate a specific subset of high-risk patients via certain pertinent risk factors so that the screening process becomes clinically and economically possible. But up until now, no guidelines have been established. Our present cohort study aims to investigate the relationship between the presence and degree of DR and silent myocardial ischemia in a diabetic population using myocardial perfusion imaging of singlephoton emission computed tomography (SPECT - MPI).

\section{Methods}

A total of 53 patients were included in a prospective cohort to define the relation between the presence and degree of diabetic retinopathy, as an independent predictor, to silent myocardial ischemia in diabetic patients. The inclusion criteria included diabetic patients with evidence of diabetic retinopathy on fundus examination, age between 30 and 65 years, and absence of ischemic symptoms like chest pain or other anginal equivalents according to Rose questionnaire [20].

\subsection{Study Design}

After an initial fundoscopic examination in mydriasis using slit-lamp biomicroscopy to detect signs of diabetic retinopathy, all enrolled patients underwent full history taking including age, residency, other comorbid diseases especially hypertension, family history of CAD, history of hypercholesterolemia, history of coronary artery disease or anginal pain, and the onset of diabetes mellitus. Then, the participants went through a clinical examination, cardiac auscultation for any abnormality, resting ECG, and OCT examination to confirm the degree of retinopathy (the worse eye was used for the evaluation) and detect additional comorbid significant diabetic macular edema (DME). Afterward, the participants were divided into two groups:

1. Mild DR group which includes patients with mild nonproliferative diabetic retinopathy (NPDR), wherein the signs of retinal affection are few microaneurysms and exudate.

2. Moderate to severe DR group which includes patients with moderate non-proliferative diabetic retinopathy (NPDR), severe non-proliferative diabetic retinopathy (NPDR), and proliferative diabetic retinopathy. Wherein the signs of retinal affection in moderate NPDR are cotton-wool spots as well as dot hemorrhages, and in severe NPDR are an eye with four quadrants with intraretinal hemorrhage, two with venous beading or one with intraretinal microvascular abnormalities (IrMAs), while the signs of PDR are the presence of neovascularization of the disc (NVD) or elsewhere (NVE) or vitreous hemorrhage [21-23].

After that, an echocardiogram was performed for all participants who then underwent stress SPECT myocardial perfusion imaging to assess the presence of myocardial ischemia; wherein the patient is considered negative upon lack of any ischemic burden or scars of the myocardial tissue, and the patient is considered positive upon detection of total reversible ischemia, partially reversible ischemia, and/or an irreversible scar.

\subsection{Imaging and Stress Protocols}

Patients were instructed to abstain from any products containing caffeine for $24 \mathrm{~h}$ before the test. Beta-blockers and calcium-channel antagonists were terminated $48 \mathrm{~h}$ before testing. Stress testing was performed with a symptom-limited Bruce treadmill exercise protocol. Twelve lead ECG was monitored continuously during stress testing. An ischemic stress ECG response was defined as $\geq 1 \mathrm{~mm}$ horizontal or down-sloping ST-segment depression or $\geq 1.5 \mathrm{~mm}$ upsloping ST depression. The full protocols have been previously described in the prior literature [24].

Stress ${ }^{201} \mathrm{Tl} /$ rest ${ }^{99 \mathrm{~m}} \mathrm{Tc}$-sestamibi Protocol: Stress ${ }^{201} \mathrm{Tl}$-/rest ${ }^{99 \mathrm{~m}}$ Tc-sestamibi imaging sequence [25] was used in all enrolled 53 patients in whom patients were injected with ${ }^{201} \mathrm{Tl}$ at peak stress. The specific weight-based dose regimen employed was to inject $74 \mathrm{MBq}(2 \mathrm{mCi})$ in patients $\leq 100 \mathrm{Kg}$ and $92.5 \mathrm{MBq}(2.5 \mathrm{mCi})$ in patients $>100 \mathrm{Kg}$. Beginning approximately $10 \mathrm{~min}$ after exercise stress, a 6 -min supine stress acquisition was performed followed by a 6 -min upright acquisition. Subsequently, in the rest phase, ${ }^{99 \mathrm{~m}} \mathrm{Tc}$-sestamibi was injected $[296 \mathrm{MBq}(8 \mathrm{mCi})$ in patients $\leq 100 \mathrm{Kg}$ and 370 $\mathrm{MBq}(10 \mathrm{mCi})$ in patients $>100 \mathrm{Kg}$ ], and beginning $2 \mathrm{~min}$ after injection, a 4-min rest upright acquisition was performed followed by a 4-min supine rest acquisition.

SPECT Scanner and Imaging Method: The system uses 9 tungsten collimated CZT detector columns rotating in synchrony, each consisting of $1024(16 \times 64)$, 5-mm thick CZT elements $(2.46 \times 2.46 \mathrm{~mm})$. The size of the collimator holes matches the dimensions of the detector elements. Before imaging, the detector was positioned parallel to the patient's chest, with the heart in the center of the field of view. A 10 -sec pre-scan acquisition was performed to identify the location of the heart and to set the angle limits of scanning for each detector column (region-of-interest centric scanning). Each image set was acquired with 120 projections per detector. Transaxial images were generated from listmode data by the use of a proprietary reconstruction algorithm based on the maximum likelihood expectation maximization method. Images were then reoriented into short-axis and vertical and horizontal long-axis slices using standard software.

Scoring System: Using a 17-segment, five-point scoring system $(0=$ normal, $1=$ equivocal, $2=$ moderate, $3=$ severe reduction of radioisotope uptake, and $4=$ absence of detectable tracer uptake) [26]; a summed stress scores were obtained by adding the scores of the 17-segments and was subsequently expressed as a percentage of abnormal myocardium at stress (SSS\% myo) [by dividing the summed maximum score $68(4 \times 17)$ and multiplying by 100] [27]. Similarly, the percentage of ischemic myocardium (SDS\% myo) was calculated by using the summed difference score 
[SSS - summed rest score].

\subsection{Statistical Analysis}

Data were fed to the computer and analyzed using IBM SPSS software package version 20.0. (Armonk, NY: IBM Corp) Qualitative data were described using the number and percent. The distribution pattern of metric discrete and continuous variables was evaluated by the KolmogorovSmirnov test. Metric discrete and continuous variables were shown as the mean \pm standard deviation (SD) or median (minimum-maximum), where applicable. The mean value differences between groups were compared by Student's ttest. Nominal data were analyzed by Pearson's chi-square or Fisher's exact test, where appropriate. Monte Carlo correction was used as a correction for chi-square when more than $20 \%$ of the cells have expected count less than 5 . Odds ratios and $95 \%$ confidence intervals for each independent variable were also calculated. A p-value of less than 0.05 was considered statistically significant.

\section{Results}

From a total of 53 diabetic patients with DR; the number of positive stress-resting $99 \mathrm{mTc}$ SestaMIBI myocardial perfusion scintigraphy was 13 cases, although no participant had wall motion abnormality on echocardiography at study entry, while the number of negative cases who have no significant perfusion defects using the same test was 40 participants (Table 1). The statistical analysis showed that diabetic retinopathy (DR) has a statistically significant relation to silent myocardial ischemia $(P=0.019)$ : wherein the prevalence of silent myocardial ischemia in the tested diabetic patients with diabetic retinopathy (regardless of the degree of DR) is $24.5 \%$.

\subsection{Mild Versus Moderate to Severe DR}

Moderate to severe DR is highly suggestive of SMI than mild DR (Odds ratio $=6.079$ with $95 \%$ confidence). Of the total 23 mild DR cases, 21 participants were negative for SMI (91.3\%) and only 2 patients suffer SMI (8.7\%). On the other hand; of the total 30 cases with moderate to severe DR, 11 patients suffer SMI (36.7\%) and 19 patients were negative to SMI $(63.3 \%)$. Moreover; Of the total of 13 cases who showed positive stress-resting myocardial perfusion scintigraphy; the percentage of patients who suffer moderate to severe DR was $84.6 \%$ in contrast to $15.4 \%$ of patients who suffer mild DR (Tables $1 \& 2$ ).

Table 1. Describing the statistical relation between DR and SMI in the tested population.

\begin{tabular}{|c|c|c|c|c|c|c|c|c|c|}
\hline \multirow{3}{*}{ DR } & \multirow{2}{*}{\multicolumn{2}{|c|}{ Total $(n=53)$}} & \multicolumn{4}{|c|}{ Silent Myocardial Ischemia } & \multirow{3}{*}{$\chi^{2}$} & \multirow{3}{*}{$\mathbf{P}^{*}$} & \multirow{3}{*}{ OR $(95 \%$ C. I) } \\
\hline & & & \multicolumn{2}{|c|}{ Negative $(n=40)$} & \multicolumn{2}{|c|}{ Positive $(n=13)$} & & & \\
\hline & No. & $\%$ & No. & $\%$ & No. & $\%$ & & & \\
\hline Mild & 23 & 43.4 & 21 & 52.5 & 2 & 15.4 & \multirow{2}{*}{5.502} & \multirow{2}{*}{0.019} & 1.000 \\
\hline Moderate to severe & 30 & 56.6 & 19 & 47.5 & 11 & 84.6 & & & $6.079(1.19-31.0)$ \\
\hline
\end{tabular}

$\chi^{2}$ : Chi-square test.

*Statistically significant at $\mathrm{p} \leq 0.05$. OR: Odds ratio. CI: Confidence interval.

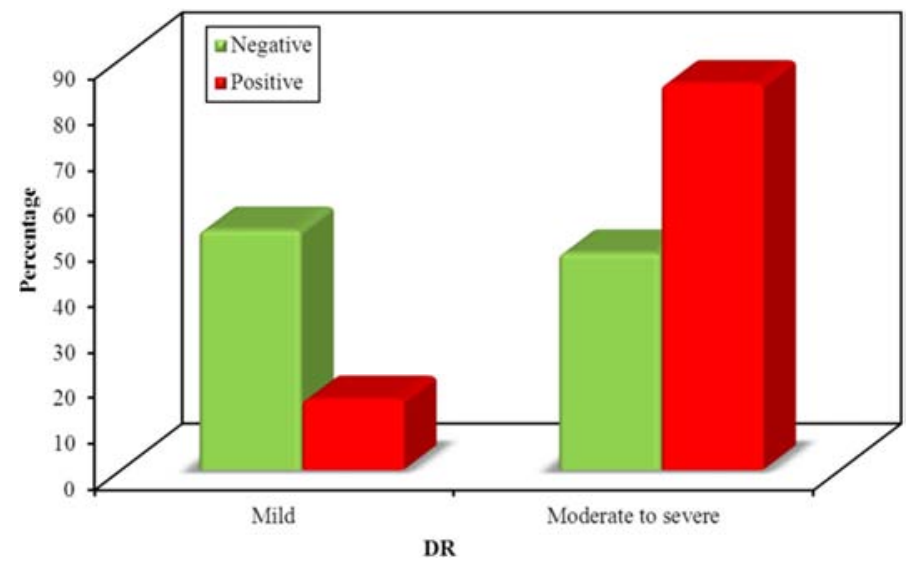

Figure 1. Showing the statistical relation between DR (according to degree) and SMI in the tested population.

Table 2. Describing the statistical relation between DR and SMI in the tested population (\% of Raw).

\begin{tabular}{|c|c|c|c|c|c|c|c|c|c|}
\hline \multirow{3}{*}{ DR } & \multirow{2}{*}{\multicolumn{2}{|c|}{ Total $(n=53)$}} & \multicolumn{4}{|c|}{ Silent Myocardial Ischemia } & \multirow{3}{*}{$\chi^{2}$} & \multirow{3}{*}{$\mathbf{P}^{*}$} & \multirow{3}{*}{ OR $(95 \%$ C. I) } \\
\hline & & & \multicolumn{2}{|c|}{ Negative $(n=40)$} & \multicolumn{2}{|c|}{ Positive $(n=13)$} & & & \\
\hline & No. & $\%$ & No. & $\%$ & No. & $\%$ & & & \\
\hline Mild & 23 & 43.4 & 21 & 91.3 & 2 & 8.7 & & & 1.000 \\
\hline Moderate to severe & 30 & 56.6 & 19 & 63.3 & 11 & 36.7 & .502 & 0.019 & $6.079(1.19-31.0)$ \\
\hline
\end{tabular}

$\chi^{2}$ : Chi-square test.

$*$ Statistically significant at $\mathrm{p} \leq 0.05$. OR: Odds ratio. CI: Confidence interval. 


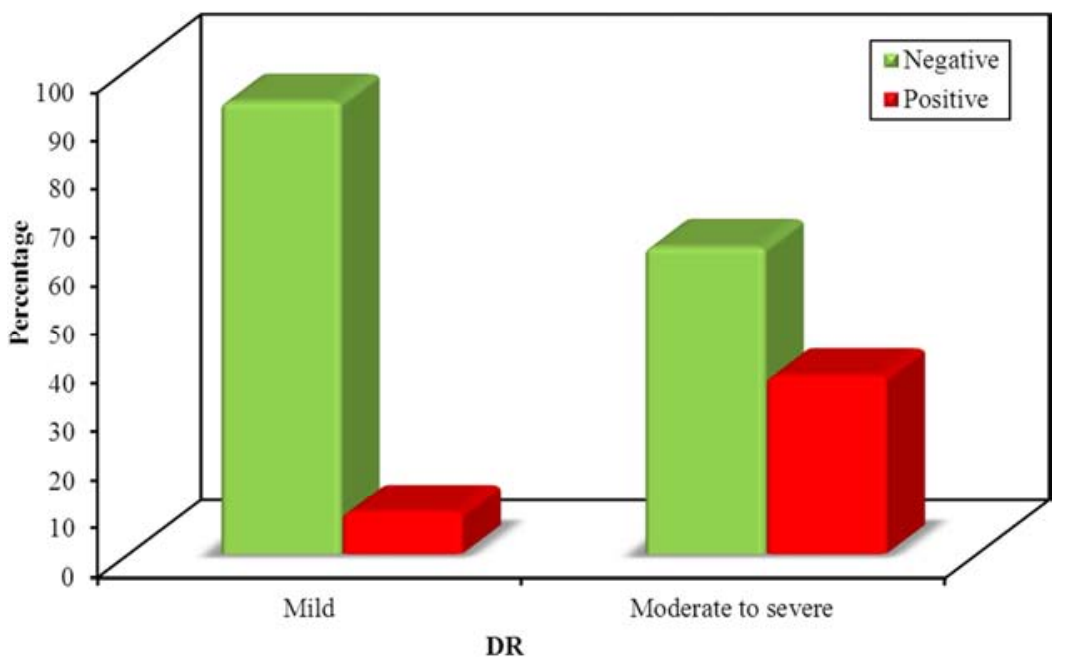

Figure 2. Showing the statistical relation between DR (according to degree) and SMI in the tested population (\% of Raw).

\subsection{Comorbidity of Hypertension}

Hypertension was a comorbid risk factor with diabetic retinopathy for silent myocardial ischemia in the tested cohort (Table $3)$, wherein hypertension has a strong statistically significant relation to silent myocardial ischemia in diabetic patients with diabetic retinopathy $(p<0.001)$.

Table 3. Describing the statistical relation between HTN and SMI in the tested population.

\begin{tabular}{|c|c|c|c|c|c|c|c|c|}
\hline & \multirow{2}{*}{\multicolumn{2}{|c|}{ Total $(n=53)$}} & \multicolumn{4}{|c|}{ Silent Myocardial Ischemia } & \multirow{3}{*}{$\chi^{2}$} & \multirow{3}{*}{$\mathbf{P *}$} \\
\hline & & & \multicolumn{2}{|c|}{ Negative $(n=40)$} & \multicolumn{2}{|c|}{ Positive $(n=13)$} & & \\
\hline & No. & $\%$ & No. & $\%$ & No. & $\%$ & & \\
\hline \multicolumn{9}{|c|}{ HTN } \\
\hline No & 28 & 52.8 & 27 & 67.5 & 1 & 7.7 & \multirow{2}{*}{14.083} & \multirow[t]{2}{*}{$<0.001$} \\
\hline Yes & 25 & 47.2 & 13 & 32.5 & 12 & 92.3 & & \\
\hline
\end{tabular}

$\chi^{2}$ : Chi-square test.

$*$ Statistically significant at $\mathrm{p} \leq 0.05$

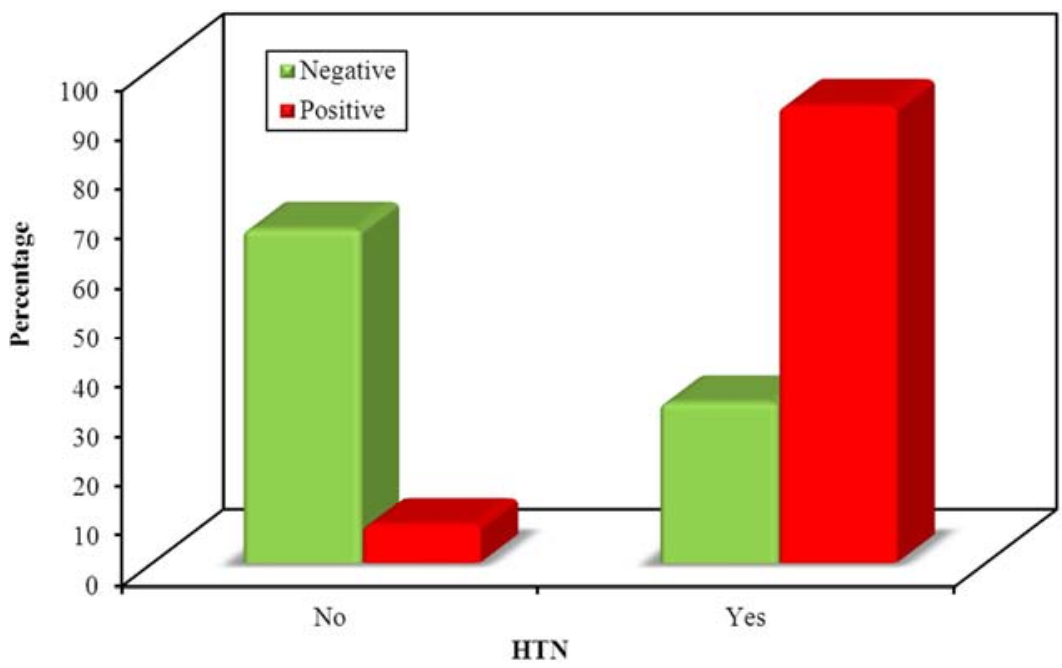

Figure 3. Showing the statistical relation between HTN and SMI in the tested population.

\subsection{Role of Diabetic Onset}

The duration of diabetes has a statistically significant relation to the presence of silent myocardial ischemia $(p<0.001)$ in the tested population (Tables $4 \& 5$ ), wherein the cutoff point of predicting silent myocardial ischemia in the tested cohort is 10 years or more (with $76.92 \%$ sensitivity and $92.5 \%$ specificity). 
Table 4. Describing the statistical relation between diabetic onset and SMI in the tested population.

\begin{tabular}{|c|c|c|c|c|c|}
\hline \multirow{2}{*}{ Onset } & \multirow{2}{*}{ Total $(n=53)$} & \multicolumn{2}{|c|}{ Silent Myocardial Ischemia } & \multirow{2}{*}{ t } & \multirow{2}{*}{$\mathbf{P}^{*}$} \\
\hline & & Negative $(n=40)$ & Positive (n=13) & & \\
\hline Min. - Max. & $3.0-17.0$ & $3.0-12.0$ & $7.0-17.0$ & & \\
\hline Mean \pm SD & $8.57 \pm 3.45$ & $7.23 \pm 2.33$ & $12.69 \pm 3.09$ & $6.776^{*}$ & $<0.001^{*}$ \\
\hline Median (IQR) & $8.0(6.0-10.0)$ & $7.0(5.50-9.0)$ & $13.0(11.0-15.0)$ & & \\
\hline
\end{tabular}

t: Student t-test.

*Statistically significant at $\mathrm{p} \leq 0.05$.

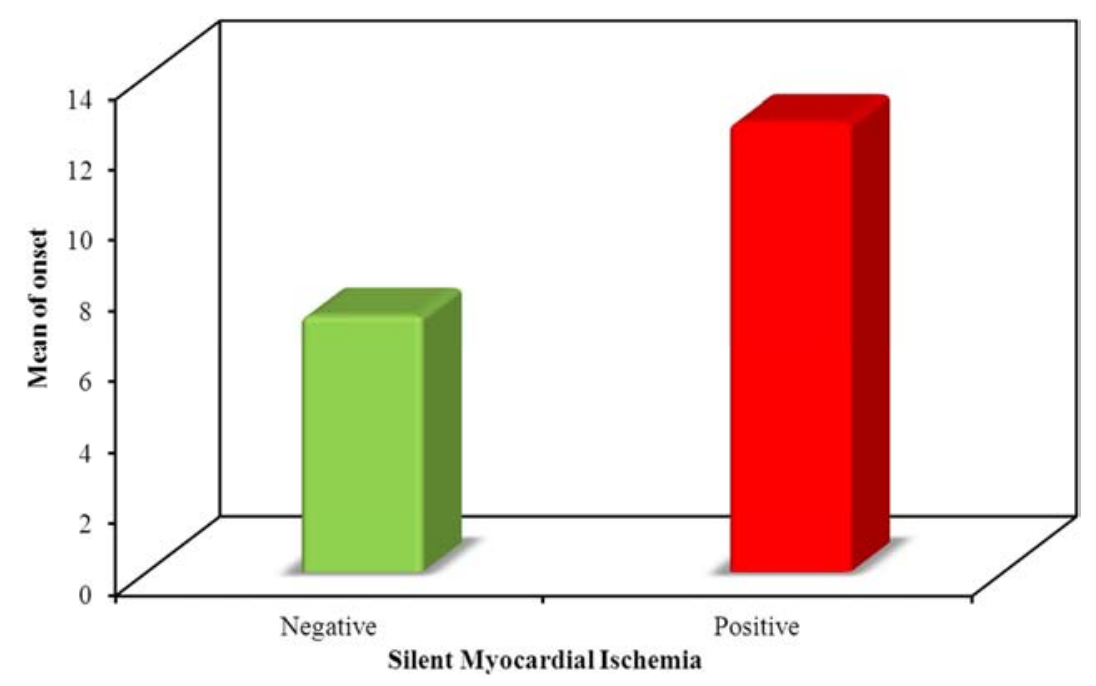

Figure 4. Showing the statistical relation between diabetic onset and SMI in the tested population.

Table 5. Describing the agreement (sensitivity, specificity) for onset to predict silent Myocardial Ischemia.

\begin{tabular}{llllll}
\hline & Cut off & Sensitivity & Specificity & PPV & NPV \\
\hline Onset & $>10$ & 76.92 & 92.50 & 76.9 & 92.5 \\
\hline
\end{tabular}

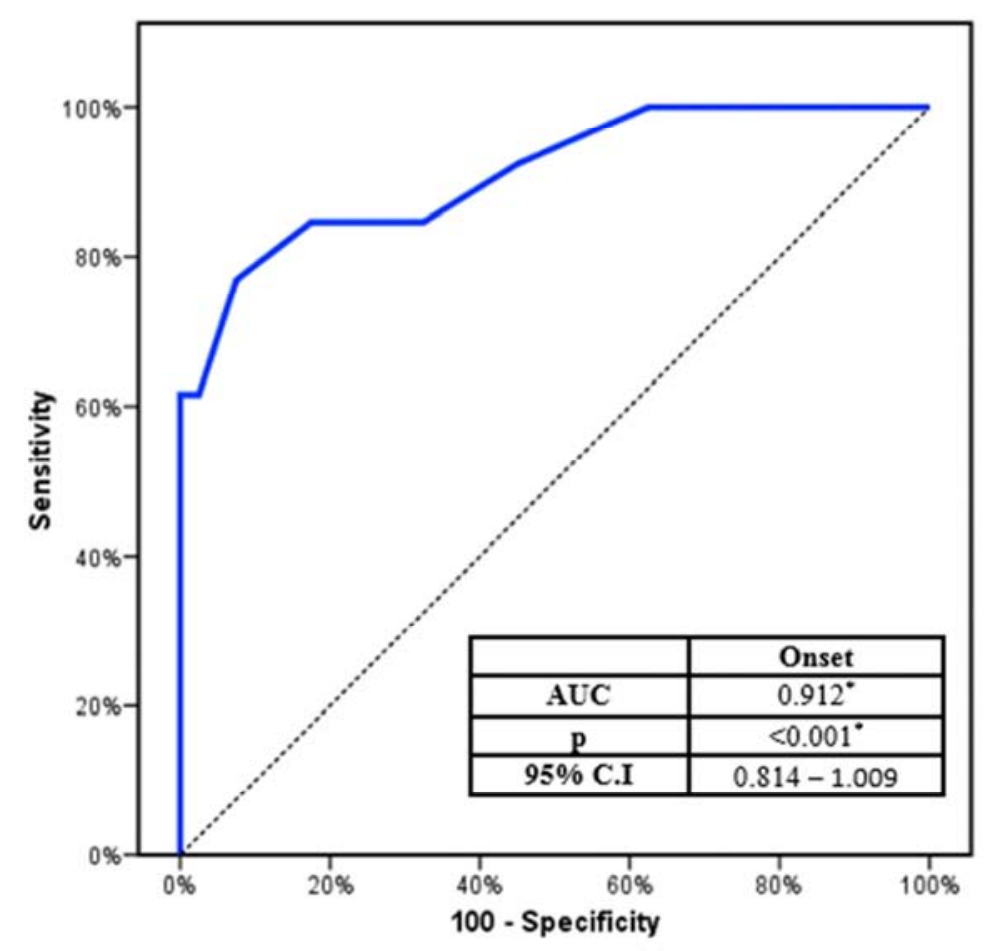

Figure 5. ROC curve for onset to predict silent Myocardial Ischemia. 


\subsection{Role of Other Tested Parameters}

Other parameters included in this cohort such as sex, age, and diabetic macular edema, do not have a statistically significant relation to silent myocardial ischemia (Tables 68). Additionally, the statistical analysis showed that the presence of silent myocardial ischemia does not affect left ventricular functional parameters: wherein the ejection fraction, end-diastolic volume and the end-systolic volume do not show statistically significant differences between the negative and positive groups.

Table 6. Describing the statistical relation between EF, EDV, ESV, and SMI in the tested population.

\begin{tabular}{|c|c|c|c|c|c|}
\hline & \multirow{2}{*}{ Total $(n=53)$} & \multicolumn{2}{|c|}{ Silent Myocardial Ischemia } & \multirow[b]{2}{*}{$\mathbf{t}$} & \multirow[b]{2}{*}{$\mathbf{p}$} \\
\hline & & Negative $(n=40)$ & Positive (n=13) & & \\
\hline \multicolumn{6}{|l|}{$\mathrm{EF}$} \\
\hline Mean \pm SD. & $64.45 \pm 5.66$ & $64.50 \pm 5.55$ & $64.31 \pm 6.21$ & \multirow{3}{*}{0.105} & \multirow{3}{*}{0.916} \\
\hline Median (IQR) & $66.0(62.0-70.0)$ & $65.50(62.5-69.5)$ & $67.0(60.0-70.0)$ & & \\
\hline EDV & & & & & \\
\hline Mean \pm SD & $78.13 \pm 22.06$ & $75.37 \pm 20.64$ & $86.62 \pm 24.94$ & \multirow{3}{*}{1.621} & \multirow{3}{*}{0.111} \\
\hline Median (IQR) & $77.0(62.0-97.0)$ & $74.50(61.50-91.0)$ & $97.0(66.0-103.0)$ & & \\
\hline ESV & & & & & \\
\hline Mean \pm SD & $27.17 \pm 11.71$ & $26.0 \pm 10.85$ & $30.77 \pm 13.90$ & \multirow{2}{*}{1.284} & \multirow{2}{*}{0.205} \\
\hline Median (IQR) & $26.0(20.0-35.0)$ & $26.0(19.50-35.0)$ & $31.0(20.0-45.0)$ & & \\
\hline
\end{tabular}

$\mathrm{t}$ : Student t-test

*Statistically significant at $\mathrm{p} \leq 0.05$.

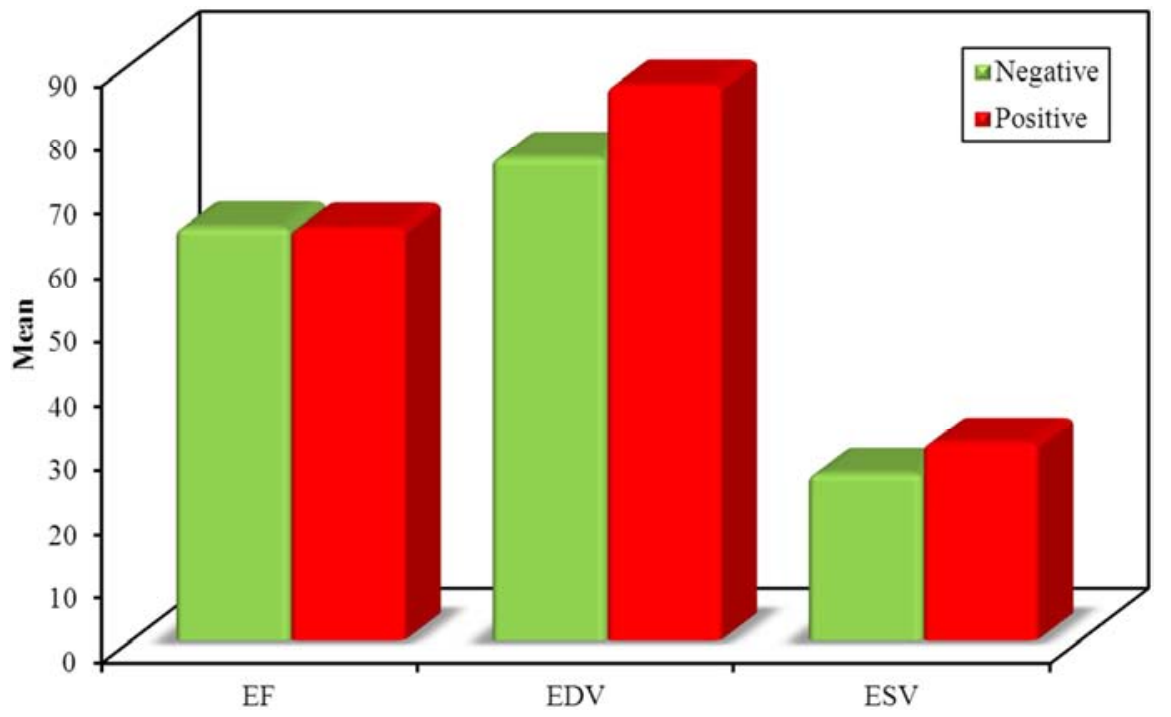

Figure 6. Showing the statistical relation between EF, EDV, ESV, and SMI in the tested population.

Table 7. Describing the statistical relation between age, sex, and SMI in the tested population.

\begin{tabular}{|c|c|c|c|c|c|c|c|c|}
\hline & \multirow{2}{*}{\multicolumn{2}{|c|}{ Total $(n=53)$}} & \multicolumn{4}{|c|}{ Silent Myocardial Ischemia } & \multirow{3}{*}{ Test of Sig. } & \multirow{3}{*}{$\mathbf{p}$} \\
\hline & & & \multicolumn{2}{|c|}{ Negative $(n=40)$} & \multicolumn{2}{|c|}{ Positive (n=13) } & & \\
\hline & No. & $\%$ & No. & $\%$ & No. & $\%$ & & \\
\hline \multicolumn{9}{|l|}{ Sex } \\
\hline Male & 26 & 49.1 & 19 & 47.5 & 7 & 53.8 & \multirow{3}{*}{$\chi^{2}=0.158$} & \multirow{3}{*}{0.691} \\
\hline Female & 27 & 50.9 & 21 & 52.5 & 6 & 46.2 & & \\
\hline Age (years) & & & & & & & & \\
\hline Min. - Max. & \multicolumn{2}{|c|}{$37.0-65.0$} & \multicolumn{2}{|c|}{$37.0-64.0$} & \multicolumn{2}{|c|}{$40.0-65.0$} & & \\
\hline Mean \pm SD. & \multicolumn{2}{|c|}{$52.58 \pm 6.76$} & \multicolumn{2}{|c|}{$52.0 \pm 6.33$} & \multirow{2}{*}{\multicolumn{2}{|c|}{$\begin{array}{l}54.38 \pm 7.95 \\
560(500-600)\end{array}$}} & $\mathrm{t}=1.107$ & 0.273 \\
\hline Median (IQR) & \multicolumn{2}{|c|}{$54.0(48.0-58.0)$} & \multicolumn{2}{|c|}{$53.0(47.50-57.0)$} & & & & \\
\hline
\end{tabular}

$\chi^{2}$ : Chi-square test t: Student t-test.

*Statistically significant at $\mathrm{p} \leq 0.05$ 


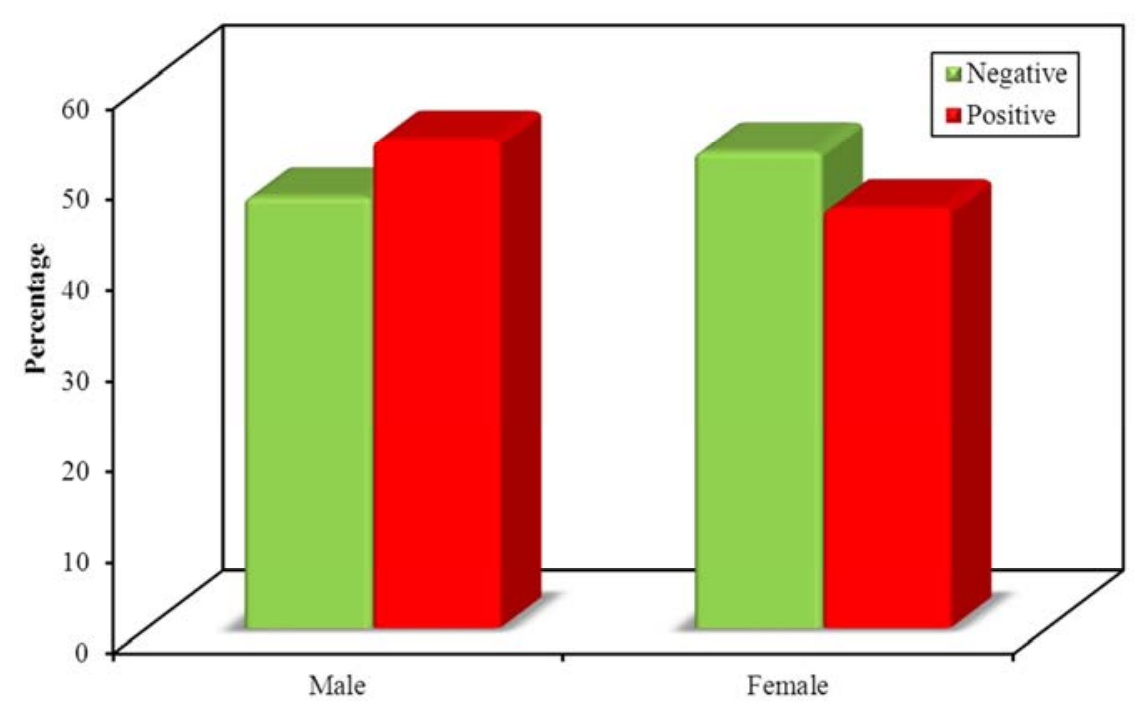

Figure 7. Showing the statistical relation between gender and SMI in the tested population.

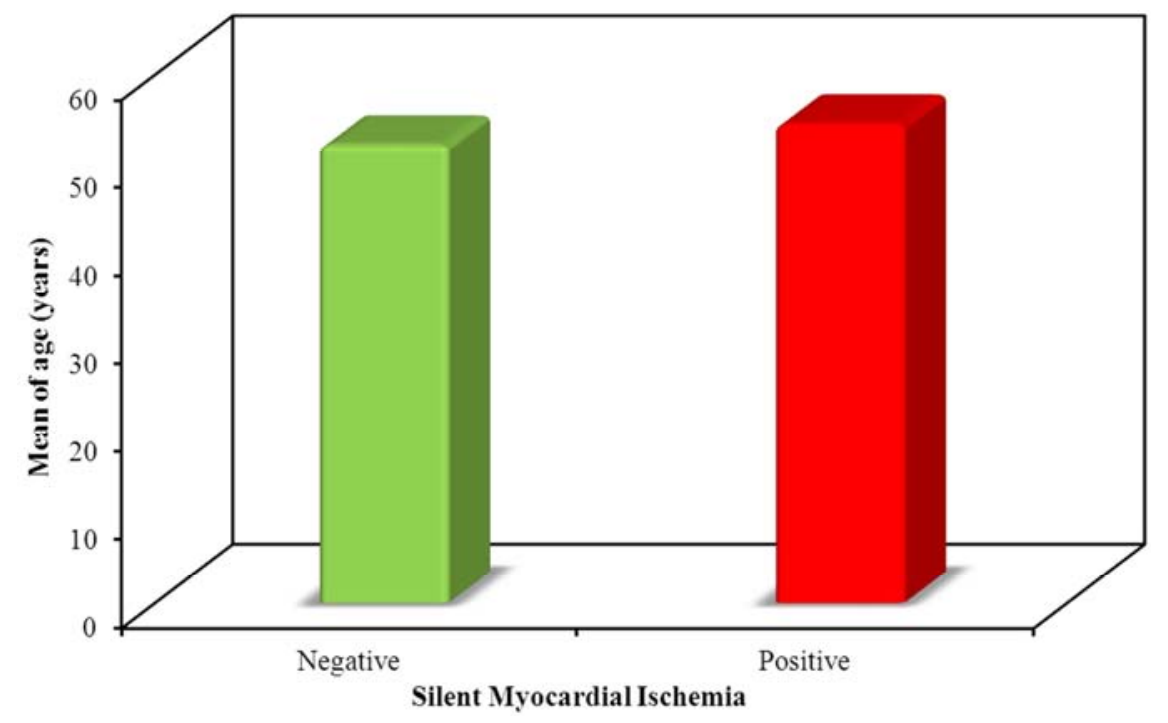

Figure 8. Showing the statistical relation between age and SMI in the tested population.

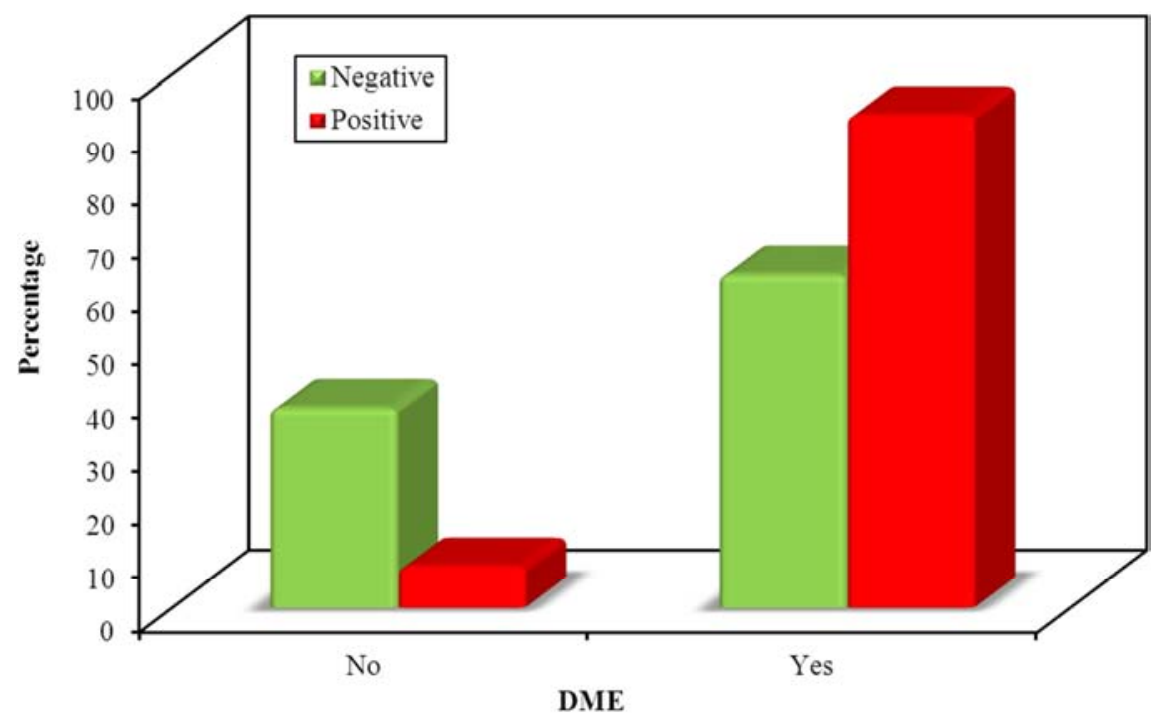

Figure 9. Showing the statistical relation between DME and SMI in the tested population. 
Table 8. Describing the statistical relation between DME and SMI in the tested population.

\begin{tabular}{|c|c|c|c|c|c|c|c|c|c|}
\hline & \multirow{2}{*}{\multicolumn{2}{|c|}{ Total $(n=53)$}} & \multicolumn{4}{|c|}{ Silent Myocardial Ischemia } & \multirow{3}{*}{$\chi^{2}$} & \multirow{3}{*}{$\mathbf{p}$} & \multirow{3}{*}{ OR (95\% C. I) } \\
\hline & & & \multicolumn{2}{|c|}{ Negative $(n=40)$} & \multicolumn{2}{|c|}{ Positive (n=13) } & & & \\
\hline & No. & $\%$ & No. & $\%$ & No. & $\%$ & & & \\
\hline DME & & & & & & & & & \\
\hline No & 16 & 30.2 & 15 & 37.5 & 1 & 7.7 & 4.136 & ${ }^{\mathrm{FE}} \mathrm{p}=0.079$ & 1.000 \\
\hline Yes & 37 & 69.8 & 25 & 62.5 & 12 & 92.3 & 4.136 & $p=0.0 / 9$ & $7.20(0.85-16.08)$ \\
\hline
\end{tabular}

$\chi^{2}$ : Chi-square test FE: Fisher Exact.

*Statistically significant at $\mathrm{p} \leq 0.05$. OR: Odds ratio. CI: Confidence interval.

\section{Discussion}

In this prospective cohort study, we analyze the relation between diabetic retinopathy and silent myocardial ischemia. Previous studies [28-36] have established such relation, however, in our study, we further investigate whether the degree of retinopathy conveys additional specific risk, besides we examine the effect of hypertension comorbidity and duration of diabetes on patient outcome regarding silent myocardial ischemia. The study aims to stratify a group of specific risk factors in a subset of diabetic patients who suffer diabetic retinopathy so that screening of said subset becomes practical, either economically or clinically, when we consider the $35 \%$ prevalence of diabetic retinopathy among diabetic patients [37, 38], which accounts for tens of millions of people in the light of the current global burden of diabetes which is 422 million, and projected to rise to 629 million by
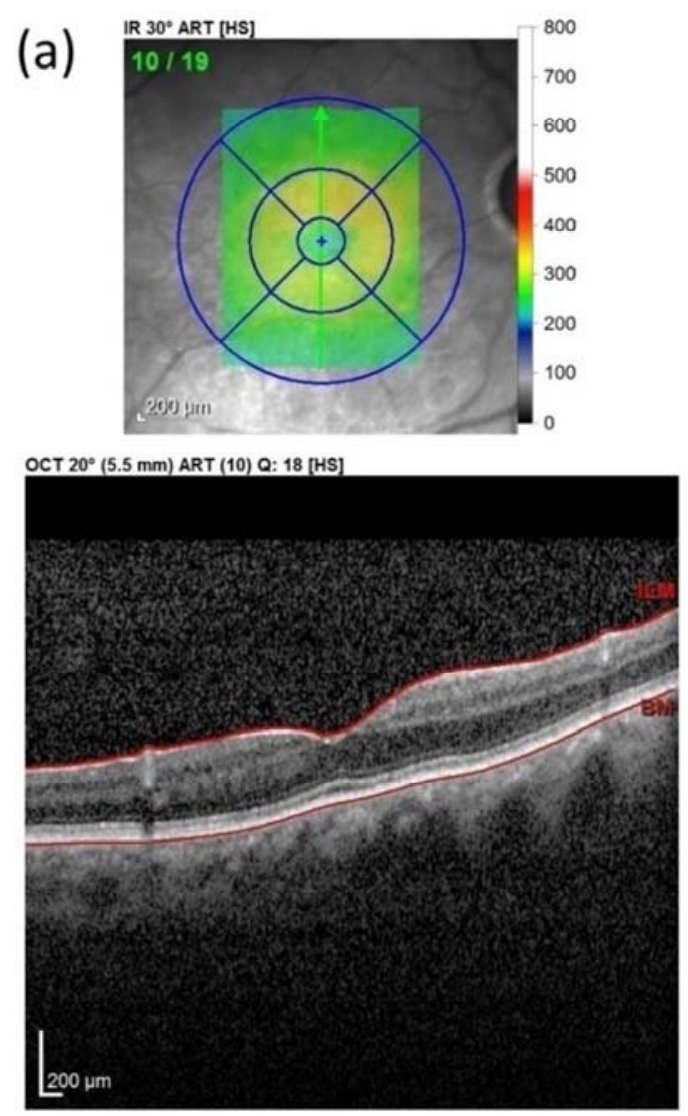

2045 [39]. In the light of this cohort, patient with moderate to severe DR is at greater risk to develop silent ischemia, especially in the presence of additional risk factor such as hypertension and/or diabetic onset $>10$ years.

The patients enrolled in this cohort study were asymptomatic diabetic patients without any clinical reason to suspect CAD. Angina was ruled out at the time of recruitment into the study with a simplified questionnaire about anginal pain or its equivalents [20] in addition to the normal resting ECG. The patients were recruited from diabetes and primary care clinics, and some patients were self-referred. The patients were on contemporary medical treatment and were under reasonable metabolic control. Yet, 13 patients $(25 \%)$ had evidence of silent myocardial ischemia in the form of myocardial perfusion defects on the scintigraphic study.
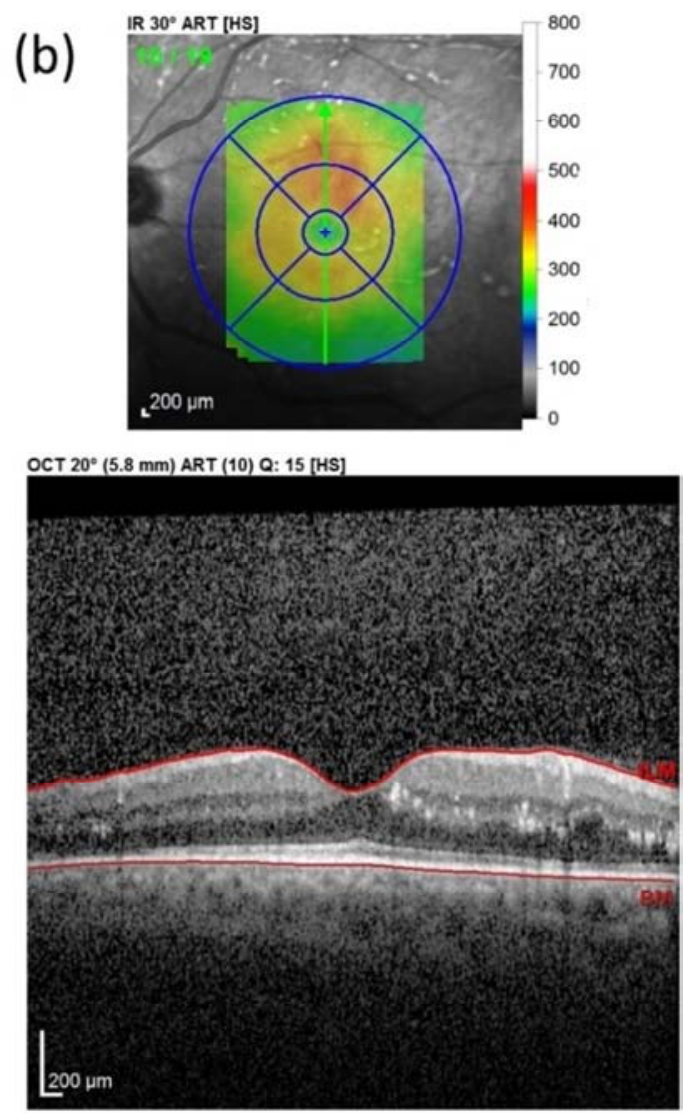

Figure 10. Two OCT images feature diabetic retinopathy; wherein (a) shows an eye affected with mild diabetic retinopathy featuring focal areas of choroidal thinning. (b) shows an eye affected with moderate to severe diabetic retinopathy featuring retinal thickening with cystic macular edema. 
Our results are consistent with other observations supporting the concept that micro and macrovascular complications of diabetes share common pathogenic mechanisms beyond those related to the classical risk factors $[1,40,41]$. Representative images of mild and moderate diabetic retinopathy detected in the OCT of the enrolled diabetic patients can be observed in Figure 10. The common pathogenic mechanisms between micro and macrovascular complications are uncertain but there is emerging evidence to suggest that DR has common genetic linkages with systemic vascular complications [42]. In addition, endothelial dysfunction, platelet dysfunction, oxidative stress, inflammation, and advanced glycation end products are pathogenic factors for both micro and macrovascular complications in diabetic patients [40, 41]. Furthermore, DR reflects widespread microcirculatory disease not only in the eye but also in vital organs elsewhere in the body such as the myocardium. Microangiopathy of the myocardium can be characterized as subendothelial and endothelial fibrosis in the coronary microvasculature leading to endothelial dysfunction that impairs myocardial blood flow reserve [43]. Therefore, retinal microvascular abnormalities may reflect an early subclinical coronary affection and predispose the development of clinical cardiovascular events [1].

Several studies supporting the proposition that DR is significantly associated with silent ischemia and increased sudden cardiovascular events [1]. Ohtomo et al concluded that approximately $20 \%$ of diabetic patients with retinopathy were found to have associated subclinical coronary artery disease [30] and also that there were no significances on other factors such as body mass index (BMI), HbAlc, eGFR, insulin usage, type of diabetes and retinopathy severity. Also, the findings of Klein et al support this premise wherein

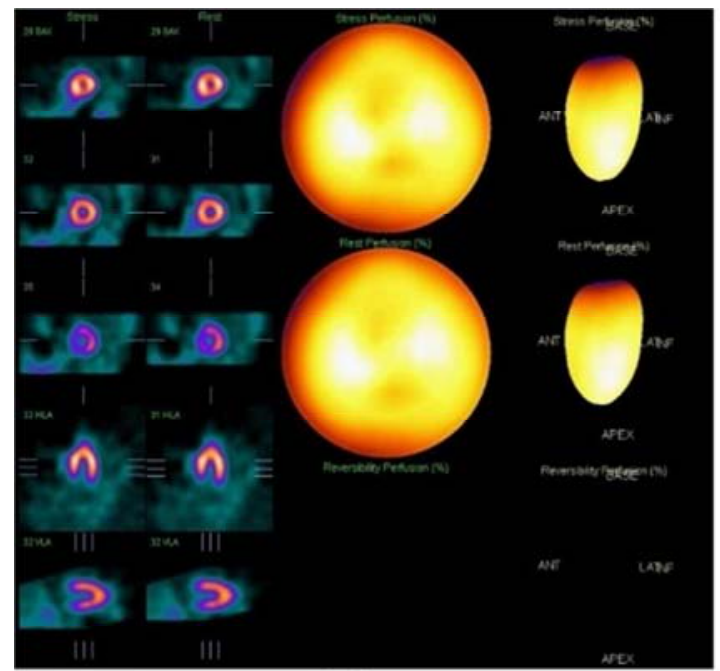

(a) carotid artery intima-media wall thickness is associated with retinopathy, but other manifestations of atherosclerosis and most of its risk factors are not associated with severity of diabetic retinopathy [44]. In contrast, the severity of diabetic retinopathy was a decisive factor in three other studies, wherein targher et al have reported in a large cohort study that diabetic retinopathy, especially in its more advanced stages, is associated with an increased incidence of ischemic heart disease independent of other known cardiovascular risk factors [4]. Um et al concluded that the degree of diabetic retinopathy correlates with the presence and severity of ischemic heart disease, wherein severe retinopathy is associated with 5-fold greater risk [36]. Also, Rong et al have reported from coronary 64-slice multi-detector computed tomography angiography analysis that the incidence and progression of DR were also associated with the severity and extent of coronary atherosclerosis [45].

Our current results are supportive of DR as a risk factor for silent myocardial ischemia, wherein 1 in each 4 tested participants had silent ischemia (SMI) with positive stressresting ${ }^{99 \mathrm{~m}}$ Tc SestaMIBI myocardial perfusion scintigraphy. Representative images of normal myocardial perfusion SPECT, mild and moderate perfusion defects detected in the enrolled diabetic patients can be observed in Figures 11 \& 12. Moreover, the degree of DR is significantly related to the incidence of affection $(P=0.019)$, wherein moderate to severe DR is highly suggestive of SMI than mild DR (Odds ratio $=6$ ). Of the total 23 mild DR cases, 21 participants were negative for SMI (91.3\%) and only 2 patients suffer SMI $(8.7 \%)$. On the other hand; of the total 30 cases with moderate to severe DR, 11 patients suffer SMI (36.7\%) and 19 patients were negative to SMI $(63.3 \%)$.

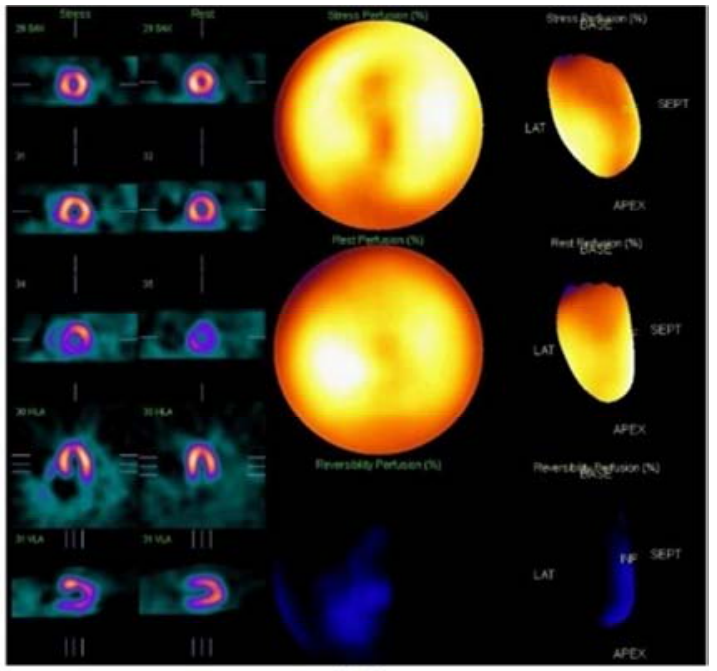

(b)

Figure 11. Two SPECT MPI (Bull's Eye View), wherein (a) showing negative Tc-99m SestaMIBI scintigraphy with normal perfusion. (b) shows a positive test with mild ischemia at the anterobasal and anterolateral segments that show reversibility at rest.

Also, it is noteworthy that Ohtomo et al used different modalities to identify silent ischemia in the enrolled group
[30]. They performed Thallium myocardial scintigraphy on only 13 cases (which account for only 7\% of the enrolled 165 
participants) from whom 4 was positive (30\%). They used the less sensitive exercise treadmill test to test 132 participants ( $80 \%$ of the enrolled population). However, the primary endpoint of their study to identify silent ischemia was the golden standard coronary angiography, but the enrollment for this CAG is still problematic due to using multiple techniques with different sensitivity. Likewise, the study performed by Klein et al was a retrospective historic cross-sectional study with regards to $\mathrm{CAD}$, wherein the authors correlated the degree of diabetic retinopathy with a history of coronary artery disease and previous strokes [44].

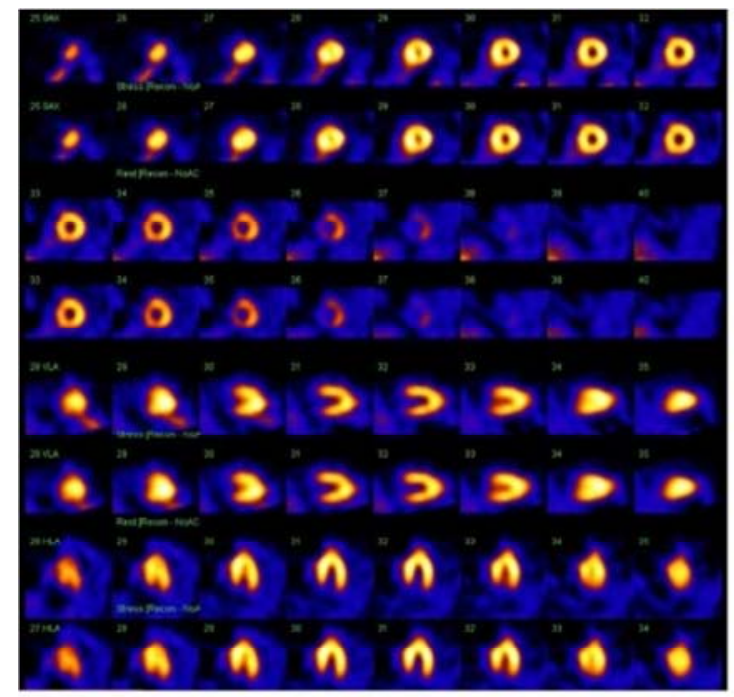

(a)
Our findings also highlight the role of hypertension and the duration of diabetes as additional risk factors that surge the incidence of silent ischemia in patients with diabetic retinopathy. The onset of DM had a statistically significant relation to silent myocardial ischemia in the tested participants $(P<0.001)$. Whereas the cutoff point of predicting silent ischemia is 10 years or more (with $76.92 \%$ sensitivity and $92.5 \%$ specificity). Also, hypertension is a comorbid risk factor as it has a strong, statically significant relation to silent myocardial ischemia in patients with diabetic retinopathy $(P<0.001)$.

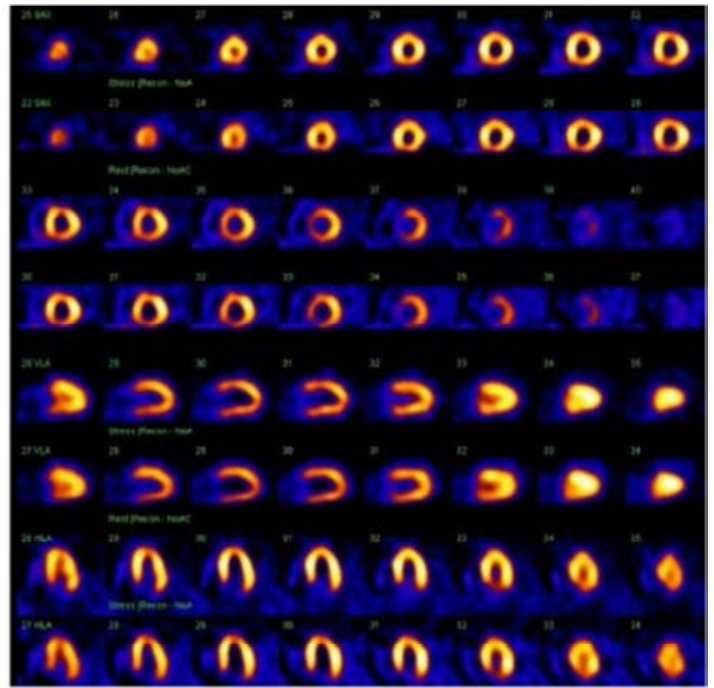

(b)

Figure 12. Two SPECT MPI (Splash View), wherein (a) showing negative Tc-99m SestaMIBI scintigraphy with normal perfusion. (b) shows a positive test with mild ischemia at the apex, antero-apical and infero-apical segments.

We also investigated the effect of silent ischemia on the functional parameters of the left ventricle such as ejection fraction, end-diastolic and end-systolic volumes. The relation was statistically insignificant $(P=0.916,0.111$ and 0.205 respectively), besides the mean values of these functional parameters were within the normal ranges, and without a considerable difference, in both positive and negative stressresting ${ }^{99 \mathrm{~m}} \mathrm{Tc}$ SestaMIBI myocardial perfusion scintigraphy (the mean $\mathrm{EF}=64.50 \pm 5.55$ in negative participants and 64.31 \pm 6.21 in positive participants, the mean $\mathrm{EDV}=75.37 \pm 20.64$ in negative participants, and $86.62 \pm 24.94$ in positive participants, the mean $\mathrm{ESV}=26.0 \pm 10.85$ in negative participants, and $30.77 \pm 13.90$ in positive participants). Hence the presence of silent myocardial ischemia does not affect the left ventricular function in the tested cohort. With regards to other variables such as gender and age; they show insignificant relation to silent ischemia ( $P=0.691$ and 0.273 respectively). Also, DME did not represent a significant risk factor for silent ischemia in the tested participants $\left(P_{F E}=0.079\right)$, in contrast with the degree of retinopathy, which is consistent with the assumption of Wong et al that diabetic patients may develop diabetic macular edema at any stage of retinopathy [46] and suggests different pathogenesis.

Nevertheless, there is a clear need for evidence-based guidelines for the early detection of coronary artery disease in the population with diabetes mellitus that depend on investigative data rather than clinical judgment. Our study indicates the important role that can be played by stress MPI in detecting silent ischemia in asymptomatic diabetic patients. Although cost-effectiveness studies are still needed; it is undeniable that diabetic patients could benefit from the identification of silent myocardial ischemia. In fact, a normal stress MPI has a very high negative predictive value for myocardial infarction or cardiac death [47]. And subjecting diabetic population with abnormal stress MPI to medical and interventional treatment after early detection of silent ischemia will essentially lead to favorable outcomes and decrease the incidence of drastic sudden cardiac events.

\section{Additional Information}

Competing Interests: The authors declare that the article was prepared in the absence of any commercial or financial relationships that could be construed as a potential conflict of interest.

\section{Abbreviations}

DR: Diabetic Retinopathy.

SMI: Silent Myocardial Ischemia. 
MPI: Myocardial Perfusion Imaging.

HTN: Hypertension.

SPECT: Single Photon Emission Computed Tomography.

OCT: Optical coherence tomography.

NPDR: Non-Proliferative Diabetic Retinopathy.

PDR: Proliferative Diabetic Retinopathy.

DME: Diabetic Macular Edema.

IrMAs: Intraretinal Microvascular Abnormalities.

NVD: Neovascularization of The Disc.

NVE: Neovascularization Elsewhere.

\section{Funding}

The authors state that this article received no funding.

\section{Ethics Approval and Consent to Participate}

The study was approved by the ethical committee of the Faculty of Medicine, Al Azhar University. Informed consent was obtained from all participants after the explanation of all the steps of the study. It was explained to all the participants that the collected data is confidential and for the purpose of scientific research only.

\section{Availability of Data and Materials}

All datasets, on which the conclusions of the manuscript rely, are presented in the main paper.

\section{References}

[1] N. Cheung, J. J. Wang, R. Klein, D. J. Couper, A. R. Sharrett, T. Y. Wong, Diabetic retinopathy and the risk of coronary heart disease: the Atherosclerosis Risk in Communities Study, Diabetes Care. 30 (2007) 1742-1746.

[2] K. Gu, C. C. Cowie, M. I. Harris, Diabetes and decline in heart disease mortality in US adults, Jama. 281 (1999) 12911297.

[3] R. S. Rosenson, P. Fioretto, P. M. Dodson, Does microvascular disease predict macrovascular events in type 2 diabetes?, Atherosclerosis. 218 (2011) 13-18.

[4] G. Targher, L. Bertolini, L. Zenari, G. Lippi, I. Pichiri, G. Zoppini, M. Muggeo, G. Arcaro, Diabetic retinopathy is associated with an increased incidence of cardiovascular events in Type 2 diabetic patients, Diabet. Med. 25 (2008) 45-50.

[5] T. Tsujimoto, H. Kajio, Y. Takahashi, M. Kishimoto, H. Noto, R. Yamamoto-Honda, M. Kamimura, M. Morooka, K. Kubota, T. Shimbo, Asymptomatic coronary heart disease in patients with type 2 diabetes with vascular complications: a crosssectional study, BMJ Open. 1 (2011) e000139.

[6] BARI Investigators, Influence of diabetes on 5-year mortality and morbidity in a randomized trial comparing $\mathrm{CABG}$ and PTCA in patients with multivessel disease. The Bypass Angioplasty Revascularization Investigation (BARI), Circulation. 96 (1997) 1761-1769.
[7] BARI Investigators, Seven-year outcome in the Bypass Angioplasty Revascularization Investigation (BARI) by treatment and diabetic status, J. Am. Coll. Cardiol. 35 (2000) $1122-1129$.

[8] M. V Van Hecke, J. M. Dekker, C. D. A. Stehouwer, B. C. P. Polak, J. H. Fuller, A. K. Sjolie, A. Kofinis, R. Rottiers, M. Porta, N. Chaturvedi, Diabetic retinopathy is associated with mortality and cardiovascular disease incidence: the EURODIAB prospective complications study, Diabetes Care. 28 (2005) 1383-1389.

[9] O. Torffvit, M. Lövestam - Adrian, E. Agardh, C. Agardh, Nephropathy, but not retinopathy, is associated with the development of heart disease in type 1 diabetes: a 12 - year observation study of 462 patients, Diabet. Med. 22 (2005) 723-729.

[10] B. E. K. Klein, R. Klein, P. E. McBride, K. J. Cruickshanks, M. Palta, M. D. Knudtson, S. E. Moss, J. O. Reinke, Cardiovascular disease, mortality, and retinal microvascular characteristics in type 1 diabetes: Wisconsin epidemiologic study of diabetic retinopathy, Arch. Intern. Med. 164 (2004) 1917-1924.

[11] M. Cusick, A. D. Meleth, E. Agrón, M. R. Fisher, G. F. Reed, G. L. Knatterud, F. B. Barton, M. D. Davis, F. L. Ferris, E. Y. Chew, Associations of mortality and diabetes complications in patients with type 1 and type 2 diabetes: early treatment diabetic retinopathy study report no. 27, Diabetes Care. 28 (2005) 617-625.

[12] C. L. Hanis, H.-H. Chu, K. Lawson, D. Hewett-Emmett, S. A. Barton, W. J. Schull, C. A. Garcia, Mortality of Mexican Americans with NIDDM: retinopathy and other predictors in Starr County, Texas, Diabetes Care. 16 (1993) 82-89.

[13] H. Miettinen, S. M. Haffner, S. Lehto, T. Rönnemaa, K. Pyörälà, M. Laakso, Retinopathy predicts coronary heart disease events in NIDDM patients, Diabetes Care. 19 (1996) $1445-1448$.

[14] M. V Van Hecke, J. M. Dekker, G. Nijpels, A. C. Moll, H. A. Van Leiden, R. J. Heine, L. M. Bouter, C. D. A. Stehouwer, B. C. P. Polak, Retinopathy is associated with cardiovascular and all-cause mortality in both diabetic and nondiabetic subjects: the hoorn study, Diabetes Care. 26 (2003) 2958.

[15] J. H. Fuller, L. K. Stevens, S. L. Wang, W. H. O. M. S. Group, Risk factors for cardiovascular mortality and morbidity: The WHO multinational study of vascular disease in diabetes., Diabetologia. 44 (2001) S54.

[16] R. Klein, B. E. K. Klein, S. E. Moss, K. J. Cruickshanks, Association of ocular disease and mortality in a diabetic population, Arch. Ophthalmol. 117 (1999) 1487-1495.

[17] R. Kawasaki, N. Cheung, F. M. A. Islam, R. Klein, B. E. K. Klein, M. F. Cotch, A. R. Sharrett, D. O’Leary, T. Y. Wong, M.-E. S. of Atherosclerosis, Is diabetic retinopathy related to subclinical cardiovascular disease?, Ophthalmology. 118 (2011) 860-865.

[18] M. J. Koistinen, Prevalence of asymptomatic myocardial ischaemia in diabetic subjects., BMJ. 301 (1990) 92-95.

[19] C. J. Ditchburn, J. A. Hall, M. de Belder, A. Davies, W. Kelly, R. Bilous, Silent myocardial ischaemia in patients with proved coronary artery disease: a comparison of diabetic and nondiabetic patients, Postgrad. Med. J. 77 (2001) 395-398. 
[20] G. Rose, P. McCartney, D. D. Reid, Self-administration of a questionnaire on chest pain and intermittent claudication., J. Epidemiol. Community Heal. 31 (1977) 42-48.

[21] Early Treatment Diabetic Retinopathy Study Research Group, Photocoagulation for diabetic macular edema, Arch Ophthalmol. 103 (1985) 1796-1806.

[22] A. Patz, R. E. Smith, The ETDRS and diabetes 2000, Ophthalmology. 98 (1991) 739-740.

[23] P. Royle, H. Mistry, P. Auguste, D. Shyangdan, K. Freeman, N. Lois, N. Waugh, Pan-retinal photocoagulation and other forms of laser treatment and drug therapies for nonproliferative diabetic retinopathy: systematic review and economic evaluation, (2015).

[24] R. Nakazato, B. K. Tamarappoo, X. Kang, A. Wolak, F. Kite, S. W. Hayes, L. E. J. Thomson, J. D. Friedman, D. S. Berman, P. J. Slomka, Quantitative upright-supine high-speed SPECT myocardial perfusion imaging for detection of coronary artery disease: correlation with invasive coronary angiography, J. Nucl. Med. 51 (2010) 1724-1731.

[25] D. S. Berman, X. Kang, B. Tamarappoo, A. Wolak, S. W. Hayes, R. Nakazato, L. E. J. Thomson, F. Kite, I. Cohen, P. J. Slomka, Stress thallium-201/rest technetium- $99 \mathrm{~m}$ sequential dual isotope high-speed myocardial perfusion imaging, JACC Cardiovasc. Imaging. 2 (2009) 273-282.

[26] D. S. Berman, H. Kiat, J. D. Friedman, F. P. Wang, K. Van Train, L. Matzer, J. Maddahi, G. Germano, Separate acquisition rest thallium-201/stress technetium-99m sestamibi dual-isotope myocardial perfusion single-photon emission computed tomography: a clinical validation study, J. Am. Coll. Cardiol. 22 (1993) 1455-1464.

[27] R. Hachamovitch, S. W. Hayes, J. D. Friedman, I. Cohen, D. S. Berman, Comparison of the short-term survival benefit associated with revascularization compared with medical therapy in patients with no prior coronary artery disease undergoing stress myocardial perfusion single photon emission computed tomography, Circulation. 107 (2003) 2900-2907.

[28] C. Hernández, J. Candell-Riera, A. Ciudin, G. Francisco, S. Aguadé-Bruix, R. Simó, Prevalence and risk factors accounting for true silent myocardial ischemia: a pilot casecontrol study comparing type 2 diabetic with non-diabetic control subjects, Cardiovasc. Diabetol. 10 (2011) 9.

[29] F. J. T. Wackers, L. H. Young, S. E. Inzucchi, D. A. Chyun, J. A. Davey, E. J. Barrett, R. Taillefer, S. D. Wittlin, G. V Heller, N. Filipchuk, Detection of Silent Myocardial Ischemia in Asymptomatic Diabetic Subjects., Diabetes Care. 27 (2004).

[30] K. Ohtomo, T. Shigeeda, A. Hirose, T. Ohno, O. Kinoshita, H. Fujita, J. Ando, R. Nagai, S. Takamoto, T. Kadowaki, Silent myocardial ischaemia in patients with diabetic retinopathy, Acta Ophthalmol. 92 (2014) e492-e493.

[31] M. Araz, Z. Celen, I. Akdemir, V. Okan, Frequency of silent myocardial ischemia in type 2 diabetic patients and the relation with poor glycemic control, Acta Diabetol. 41 (2004) $38-43$.

[32] W. T. O’Neal, K. E. Lee, E. Z. Soliman, R. Klein, B. E. K. Klein, Predictors of electrocardiographic abnormalities in type 1 diabetes: the Wisconsin Epidemiologic Study of Diabetic Retinopathy, J. Endocrinol. Invest. 40 (2017) 313-318.
[33] G. Al-Humaidi, I. Sarikaya, A. H. Elgazzar, A. Owunwanne, Myocardial perfusion abnormalities in asymptomatic type 2 diabetic patients, J. Saudi Hear. Assoc. 30 (2018) 3-8.

[34] P. Valensi, A. Avignon, A. Sultan, B. Chanu, M. T. Nguyen, E. Cosson, Atherogenic dyslipidemia and risk of silent coronary artery disease in asymptomatic patients with type 2 diabetes: a cross-sectional study, Cardiovasc. Diabetol. 15 (2016) 104.

[35] S. V. de Kreutzenberg, A. Solini, E. Vitolo, A. Boi, S. Bacci, S. Cocozza, R. Nappo, A. Rivellese, A. Avogaro, M. G. Baroni, Silent coronary heart disease in patients with type 2 diabetes: Application of a screening approach in a follow-up study, J. Diabetes Complications. 31 (2017) 952-957.

[36] T. Um, D. H. Lee, J.-W. Kang, E. Y. Kim, Y. H. Yoon, The degree of diabetic retinopathy in patients with type 2 diabetes correlates with the presence and severity of coronary heart disease, J. Korean Med. Sci. 31 (2016) 1292-1299.

[37] R. Cheloni, S. A. Gandolfi, C. Signorelli, A. Odone, Global prevalence of diabetic retinopathy: protocol for a systematic review and meta-analysis, BMJ Open. 9 (2019) e022188.

[38] J. W. Y. Yau, S. L. Rogers, R. Kawasaki, E. L. Lamoureux, J. W. Kowalski, T. Bek, S.-J. Chen, J. M. Dekker, A. Fletcher, J. Grauslund, Global prevalence and major risk factors of diabetic retinopathy, Diabetes Care. 35 (2012) 556-564.

[39] B. Zhou, Y. Lu, K. Hajifathalian, J. Bentham, M. Di Cesare, G. Danaei, H. Bixby, M. J. Cowan, M. K. Ali, C. Taddei, Worldwide trends in diabetes since 1980: a pooled analysis of 751 population-based studies with $4 \cdot 4$ million participants, Lancet. 387 (2016) 1513-1530.

[40] A. Juutilainen, S. Lehto, T. Rönnemaa, K. Pyörälä, M. Laakso, Retinopathy predicts cardiovascular mortality in type 2 diabetic men and women, Diabetes Care. 30 (2007) 292-299.

[41] M. Rema, V. Mohan, R. Deepa, R. Ravikumar, Association of carotid intima-media thickness and arterial stiffness with diabetic retinopathy: the Chennai Urban Rural Epidemiology Study (CURES-2), Diabetes Care. 27 (2004) 1962-1967.

[42] N. Cheung, T. Y. Wong, Diabetic retinopathy and systemic vascular complications, Prog. Retin. Eye Res. 27 (2008) 161176.

[43] S. Moir, L. Hanekom, Z. Y. Fang, B. Haluska, C. Wong, M. Burgess, T. H. Marwick, Relationship between myocardial perfusion and dysfunction in diabetic cardiomyopathy: a study of quantitative contrast echocardiography and strain rate imaging, Heart. 92 (2006) 1414-1419.

[44] R. Klein, A. R. Sharrett, B. E. K. Klein, S. E. Moss, A. R. Folsom, T. Y. Wong, F. L. Brancati, L. D. Hubbard, D. Couper, A. Group, The association of atherosclerosis, vascular risk factors, and retinopathy in adults with diabetes: the atherosclerosis risk in communities study, Ophthalmology. 109 (2002) 1225-1234.

[45] J. Rong, C.-Q. Yu, P. Yang, J. Chen, Association of retinopathy with coronary atherosclerosis determined by coronary 64 -slice multidetector computed tomography angiography in type 2 diabetes, Diabetes Vasc. Dis. Res. 10 (2013) 161-168.

[46] T. Y. Wong, C. M. G. Cheung, M. Larsen, S. Sharma, R. Simó, Diabetic retinopathy, Nat. Rev. Dis. Prim. 2 (2016) 16012. https://doi.org/10.1038/nrdp.2016.12. 
[47] L. D. Metz, M. Beattie, R. Hom, R. F. Redberg, D. Grady, K.

E. Fleischmann, The prognostic value of normal exercise myocardial perfusion imaging and exercise echocardiography: a meta-analysis, J. Am. Coll. Cardiol. 49 (2007) 227-237. 